\section{Identifying the main risk factor for falling in community-dwelling older adults}

L Galhardas', H Sardica', A Bento', F Mendes², J Bravo ${ }^{1}$, C Pereira' ${ }^{1}$ Departamento de Desporto e Saúde, Escola de Ciências e Tecnologia, Universidade de Évora, Portugal

${ }^{2}$ Escola Superior de Enfermagem São João de Deus, Universidade de Évora, Portugal

Introduction:

Injuries resulting from falls are a major problem facing older adults. Several studies showed that falls may occur due to limitations in functional fitness, poor body composition, or ognitive and emotional factors.

\section{Objectives:}

The aim of this study was to identify preponderant physical fitness, body composition, cognitive and emotional risk factors for falls in community-dwelling older adults.

\section{Methods:}

A total of 515 people aged 65 and over residents in the Alentejo, accepted to take part of this study (114 men). Physical fitness was evaluated using the Senior Fitness Test. Each participant body fat mass weight and body mass index were assessed by bioimpedance. Geriatric depression was assessed by Geriatric Depression Scale and cognitive impairment by Mini-Mental State Examination. The collected data were analysed with SPSS (v. 22). Univariate and multivariate binary logistic regression were used to identify the variables that significantly explained fall occurrence. Receiver operating characteristic curve (ROC) was used to compute the area under the curve (AUC) for the multivariate regression model with the selected preponderant risk factors explaining falls occurrence

\section{Results:}

Univariate analysis showed that almost all studied variables explained falls occurrence, $\mathrm{p}<0.05$. Nonetheless, the variables selected by multivariate modelling as predominant risk factors for falls were gender (Male OR: 0.551, CI 95\%: $0.350-0.867$ ), lower limbs strength (OR: 0.955 CI 95\%: 0.936. - 0.975) and depression level (OR: 1.104, CI 95\%: 1.052 - 1.159); with an AUC of 0.643, CI 95\%: $0.595-0.692$

\section{Conclusions:}

Decreased lower limbs strength and increased depression levels showed to be modified preponderant risk factor for falling. Intervention for falls prevention shall evaluate these variables for individual fall risk assessment and privilege the improvement of these variables, particularly in women since results evidenced that they are almost twice more susceptive for falling than man. 